\title{
Cystic fibrosis carrier screening: Issues in implementation
}

The transition of testing in the cystic fibrosis transmembrane conductance regulator (CFTR) gene to a prenatal and preconceptional carrier screening program ${ }^{1,2}$ has been both interesting and informative. The long-held sense in the medical genetics community that there is significant genetic variability in the population has been reinforced as more people are tested and we continue to learn more. It is also apparent that there are varying perspectives about how testing should be done and how results should be communicated, as well as about ongoing program updates and education of providers and patients.

In 1997, a National Institutes of Health Consensus Development Conference ${ }^{3}$ recommended the implementation of prenatal and preconceptional carrier screening for cystic fibrosis (CF). However, it was immediately clear that laboratory testing for CFTR gene mutations and variations was not standardized and that few educational materials to support such a new program were available to providers on the front lines (obstetricians and family physicians) and their patients. A workshop in 1998 was the genesis of a collaborative effort involving the American College of Medical Genetics (ACMG), the American College of Obstetricians and Gynecologists (ACOG), and the National Human Genome Research Institute's Ethical, Legal and Social Implications program to develop the materials and standards needed to ensure the appropriate implementation of this program. Over the next 2 years, ACMG used existing CF patient and family data to identify a set of mutations that should be a core component of a screening program. ACOG, in collaboration with the National Human Genome Research Institute, worked to develop educational materials for providers and patients. The program was announced in September $2001 .^{2}$

At the time the mutation panel was set, it was clear that the inherent variation between ethnic groups in both disease incidence and the clinical sensitivity of the panel was dramatic. For the ACMG mutation panel, this is apparent in updated data from a Centers for Disease Control and Prevention-sponsored study (www.cdc.gov/genomics/info/reports/research/FBR/ ACCE.htm) showing the differences between non-Hispanic Caucasians ( $\mathrm{CF}$ incidence $=1 / 2,500)$ and Chinese Americans (CF incidence 1/31,000). Inasmuch as more data come from the ethnic groups most affected, it is apparent that the panel is going to best represent the spectrum of mutations in those groups, and this contributes to the differences in clinical sensitivity in various ethnic groups (e.g., Ashkenazim carrier detection rate $=97 \%$ versus 56\% in Hispanic Americans). Furthermore, data were based on a population defined by a disease for which money was most expended for basic research and for clinical testing, with the long-term clinical investigative work being done in the course of service provision. Some information on CF carrier screening in a prenatal diagnostic setting was available. $^{3-8}$ However, little genetics research money is expended on the "normal" or general population; its genetic characteristics remain poorly understood. CF represents a classic example of the way genetic information evolves.

Genetic testing typically begins with the inherent biases of gene identification studies in a group with the most classic and severe presentations. It then moves to studies within these families that are often biased by similar genetic backgrounds on which mutations sit that can account for the extreme presentations in the initial patients and their families. However, intrafamilial variation begins to break down many of the most extreme biases. Studies progress to less severe presentations of the condition under consideration, specific phenotypes, or other factors that identify higher-risk groups (e.g., ethnicity). The last phase involves very large or general population studies that ultimately provide the unbiased perspective. When a test is considered part of a public health activity, it is usually pilottested with state-supported funding to accumulate a body of data about the particular genetic disease marker in a general population. However, these uses are generally aimed only toward the diagnosis of affected individuals. Although some general population data were available for the CFTR gene, very large, general population studies for most genes have not been among the beneficiaries of such support and are left to evolve in the private sector marketplace environment. Another side result of these services being predominantly in the health care marketplace rather than in public health programs is that the full breadth of a screening program must evolve as well. Many of the required ancillary services were highlighted in the announcement of the screening program. Many require additional support to fully evolve.

The initial CFTR mutation and variant panel was determined on the basis of the prevalence of mutations in more than 20,000 classical CF patients, ${ }^{1}$ a seemingly large, although geographically diverse, population. Any mutation representing $0.1 \%$ or more of CFTR alleles in a pan-ethnic population was included. This resulted in 25 mutations and 4 variants known to modify the expression of one of the mutations. Insufficient data on specific ethnic groups were available and the inherent complexity of targeting screening to specific ethnic groups resulted in the initial panel's being limited and pan-ethnic.

In the ensuing year since implementation, both anticipated and new problems have been recognized and a somewhat typical course of adoption of the recommendations has been experienced. CFTR mutation testing has increased by as much as 
5-fold in large private sector laboratories and continues to increase.

Among the general problems that were anticipated were those related to the significant variability among humans and those deriving from the unequal distribution of data among ethnic groups. In CF, this is seen at the level of disease incidence variation overlaid with variability in the distribution of mutations among ethnic groups. We discuss below some of the issues that have developed with the implementation of CFTR mutation screening now that large amounts of general population data are available. This information should allow for providers to improve their communication of test results and provide the basis for further modification of the core mutation panel, as needed.

\section{$5 T / 7 T / 9 T$}

The 5T/7T/9T variant in intron 8 (IVS-8) modifies the expression of some CFTR mutations and, therefore, is used as a reflex test to determine whether particular mutations are likely to be associated with a CF phenotype. However, some laboratories have included testing of this marker as primary target. The $\mathrm{R} 117 \mathrm{H}$ mutation and its association with the $5 \mathrm{~T}$ polymorphism was appreciated at the time the carrier screening mutation panel was developed and was discussed in some detail. When $5 \mathrm{~T}$ is paired with another CFTR mutation, it can be associated with the congenital bilateral absence of the vas deferens (CBAVD) type of male infertility. The $7 \mathrm{~T}$ variant can also be found in CBAVD when paired with the R117H mutation. Similarly, the 5T mutation, when paired in a trans configuration with $\mathrm{R} 117 \mathrm{H}$, has been found in men with CBAVD. When $\mathrm{R} 117 \mathrm{H}$ is paired with $5 \mathrm{~T}$ in a cis configuration and another CF mutation is present, it is associated with a variable CF phenotype. Complicating the testing for $5 \mathrm{~T}$ are two main issues. First, $5 \mathrm{~T}$ is a very common polymorphism, being found in about $5 \%$ of the general population, and $5 \mathrm{~T}$ homozygosity has been associated with CBAVD. Furthermore, 5T homozygosity or $\Delta \mathrm{F} 508 / 5 \mathrm{~T}$ has been reported in rare cases of adult-onset CF-like pulmonary disease, though on a background of TG12M470 V that complicates its interpetation. ${ }^{9}$ However, because the goal of the screening program was to identify individuals at risk for classical CF, not CBAVD, it was recommended that $5 \mathrm{~T}$ status only be reflexly tested or reported in a parent demonstrated to have the R117H mutation. Furthermore, prenatal testing to consider risk for offspring with CF based on the presence of 5T alone is not justified.

A major contributing factor for confusion in testing for $5 \mathrm{~T}$ is that some of the technologies that allow high-volume testing for CFTR at low cost attain this goal only by maximizing the number of gene sequence targets included in a single test. Hence, testing for 5T in some systems is an all-or-none choice. This has led some laboratories to include 5T genotyping in the first-tier mutation panel, which is counter to the intent of the recommendations. When the variant is found, the laboratory is left with a difficult reporting dilemma. Although "testing panels" are increasingly frowned upon by payers, genetic panels can be quite different from the classic panels that carried unrelated analytes and initially raised concerns. Genetic test panels appropriately include multiple gene sequences from single genes that reflect population variation or multiple genes that cause the same condition. Efforts continue to maintain high standards for testing laboratories as new technologies are used in CFTR mutation testing. ${ }^{10}$

\section{I148T}

The I148T mutation was included in the panel on the basis of its prevalence of $0.1 \%$ in CF patients. However, because of its relative rarity, little was known about its occurrence in a general population. At the time the panel was determined, there were one or two anecdotal reports of individuals with I148T but without CF in families in which there were individuals with CF and I148T. After the introduction of general population carrier screening, it was observed that the I148T mutation was appearing at rates 60 to 100 times above that expected based on the rate in the CF patient population (E.M. Rohlfs, personal communication, 2002). ${ }^{11-14}$ This suggested that there was likely a modifier of the expression of I148T or that I148T was modifying the effects of another mutation. Rohlfs et al. ${ }^{13}$ recently reported that $\mathrm{I} 148 \mathrm{~T}$ segregates on at least two genetic backgrounds distinguished by the 5T/7T/9T polymorphism and a CFTR deletion, 3199del6. Among five unaffected individuals with I148T and the $\Delta \mathrm{F} 508$ or another I148T mutation, all had I148T on a 9T background. Among five individuals with CF and with $\Delta$ F508 or N1303K mutation and I148T, four had I148T on a background of 9T and 3199del6.

More cases with I148T on the 3199del6 background need to studied and predictive values established. In the meantime, when I148T is found in patients with CF, the haplotype background should be tested as a reflex test. When found in a carrier screening program, care should be taken communicating results and laboratories should consider reflex testing to determine the genetic background on which I148T sits. However, it is recognized that this new mutation is not included in many currently available testing panels. Providers should remain aware of developments specific to this mutation.

In summary, care will have to be taken in the way certain genetic changes are interpreted, and many may require subsequent or simultaneous testing to clarify their implications for those tested. The $5 \mathrm{~T}$ polymorphism should be treated as a reflex when used in carrier screening to reduce the negative impacts on both patients and providers. I148T represents an example of a genetic change that requires follow-up testing to fully understand its implications. Attention to the evolving literature related to these and other genetic changes associated with CFTR carrier screening will have to be maintained, and appropriate expertise in interpreting and communicating results will need to be available to the primary providers of these services. At the present time, the panel has been in use for nearly 1 year. Plans are already in place among the groups involved in development of the program for a comprehensive review and evaluation of current data on CFTR mutations in 
CF patients and in the general population to assess the structure of the panel itself and to evaluate problems in the implementation of carrier screening.

Michael S. Watson, PhD Washington University School of Medicine St. Louis, Missouri

Robert J. Desnick, MD, PhD Mount Sinai School of Medicine New York, New York

Wayne W. Grody, MD, PhD UCLA School of Medicine Los Angeles, California

Michael T. Mennuti, MD University of Pennsylvania Philadelphia, Pennsylvania

Bradley W. Popovich, PhD Xenon Genetics Burnaby, British Columbia, Canada

C. Sue Richards, PhD Baylor College of Medicine Houston, Texas

\section{References}

1. Grody WW, Cutting GR, Klinger KW, Richards CS, Watson MS, Desnick RJ. Laboratory standards and guidelines for population-based cystic fibrosis carrier screening. Genet Med 2001;3:149-154.
2. American College of Obstetricians and Gynecologists and American College of Medical Genetics. Preconception and prenatal carrier screening for cystic fibrosis: Clinical and laboratory guidelines. Washington, DC: American College of Obstetricians and Gynecologists, 2001.

3. Genetic testing for cystic fibrosis. National Institutes of Health Consensus Development Conference Statement on genetic testing for cystic fibrosis. Arch Intern Med 1999;159:1529-1539.

4. Doherty RA, Palomaki GE, Kloza EM, Erickson JL, Haddow JE. Couple based prenatal screening for cystic fibrosis in primary care settings. Prenat Diagn 1996;16: 397-404.

5. Grody WW, Dunkel-Schetter C, Tanagawa ZH, Fox MA, Fang CY, Cantor RM, Novak JM, Bass HN, Crandall BF. PCR-based screening for cystic fibrosis carrier mutations in an ethnically diverse pregnant population. Am J Hum Genet 1997;60: 935-937.

6. Schwartz M, Brandt NJ, Skovby F. Screening for carriers of cystic fibrosis among pregnant women: a pilot study. Eur J Hum Genet 1993;1:239-244.

7. Mennie ME, Gilfillan A, Compton M, Curtis L, Liston WA, Pullen I, Whyte DA, Brock DJ. Prenatal screening for cystic fibrosis. Lancet 1992;340:214-216.

8. Miedzybrodzka ZH, Yin Z, Kelly KF, Haites NE. Evaluation of laboratory methods for cystic fibrosis carrier screening: reliability, sensitivity, specificity, and costs. J Med Genet 1994;31:545-550.

9. Noone PG, Pue CA, Zhou Z, Friedman KJ, Wakeling EL, Ganeshananthan M, Simon RH, Silverman LM, Knowles MR. Lung disease associated with the IVS8 5 T allele of the CFTR gene. Am J Respir Crit Care Med 2000;162:1919-1924.

10. Richards CS, Bardley LA, Amos J, Allitto B, Grody WW, Maddalena A, McGinniss MJ, Prior TW, Popovich BW, Watson MS. Standards and guidelines for CFTR mutation testing. Genet Med 2002;4:379-391.

11. Loader S, Caldwell P, Kozyra A, Levenkorn JC, Boehm CD, Kazazian HH, Rowley PT. Cystic fibrosis carrier population screening in the primary care setting. Am J Hum Genet 1996;59:234-247.

12. Heim RA, Sugarman EA, Allitto BA. Improved detection of cystic fibrosis mutation in the heterogeneous US population using an expanded, pan-ethnic mutation panel. Genet Med 2001;3:168-176.

13. Rohlfs EM, Zhou Z, Sugarman EA, Heim RA, Pace RG, Knowles MR, Silverman LM, Allitto BA. The I148T CFTR allele occurs on multiple haplotypes: a complex allele is associated with cystic fibrosis. Genet Med 4:319-323.

14. Strom CM, Huang D, Buller A, Redman J, Crossley B, Anderson B, Entwistle T, Sun W. Cystic fibrosis screening using the College panel: platform comparison and lessons learned from the first 20,000 samples. Genet Med 2002;4:289-296. 